Supplement of Saf. Nucl. Waste Disposal, 1, 227-229, 2021

https://doi.org/10.5194/sand-1-227-2021-supplement

(C) Author(s) 2021. CC BY 4.0 License.

Supplement of

\title{
Specific cultural and political challenges in cross-border public participation
}

\section{Franziska Sperfeld and Melanie Mbah}

Correspondence to: Franziska Sperfeld (franziska.sperfeld@ufu.de)

The copyright of individual parts of the supplement might differ from the article licence. 


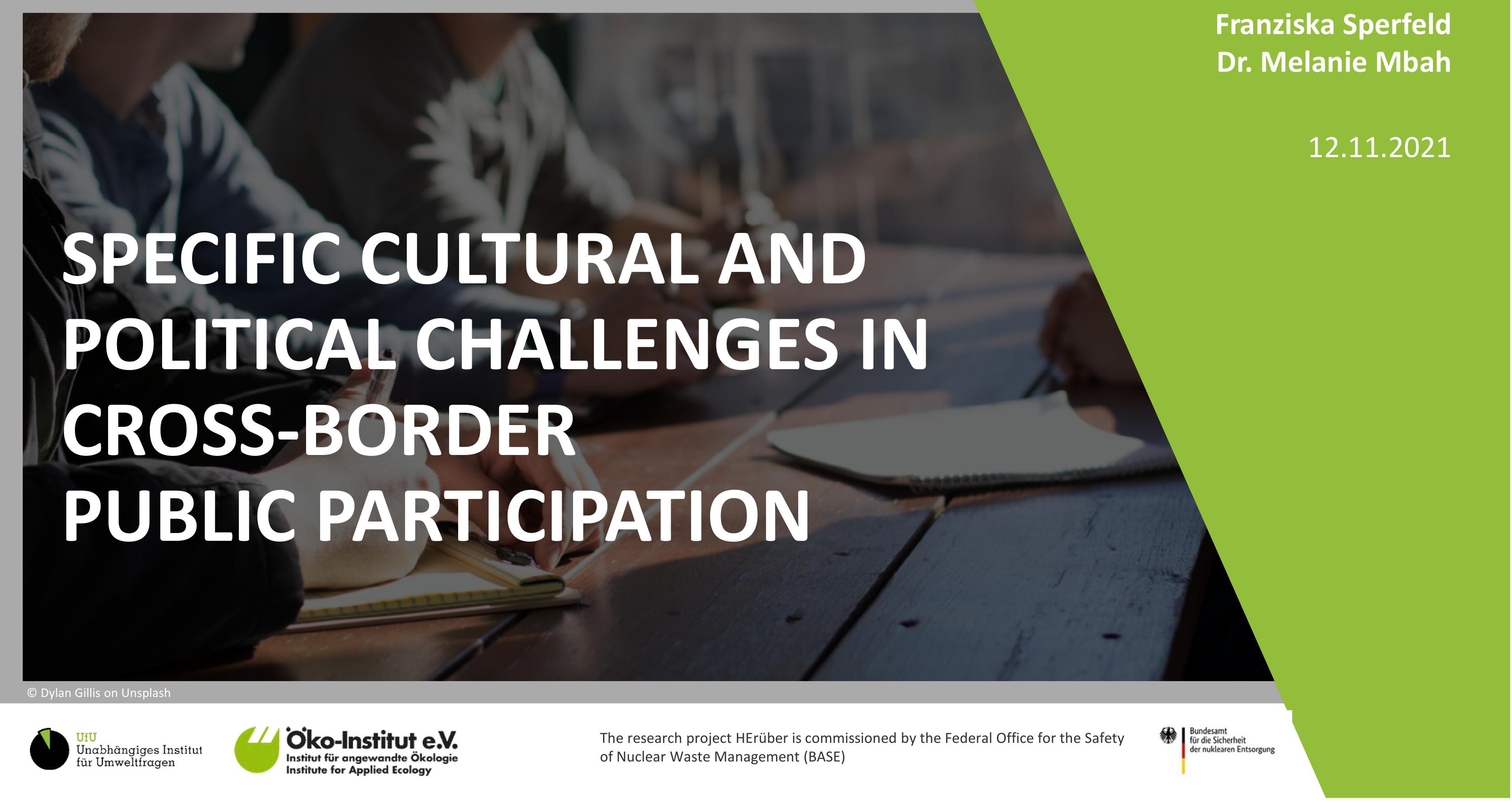




\section{First results from a} literature review on crossborder public participation

1. Research project HErüber

2. Challenges and success factors for cross-border public participation procedures

3. Specific cultural and political framework conditions in neighbouring countries

4. Outlook

西




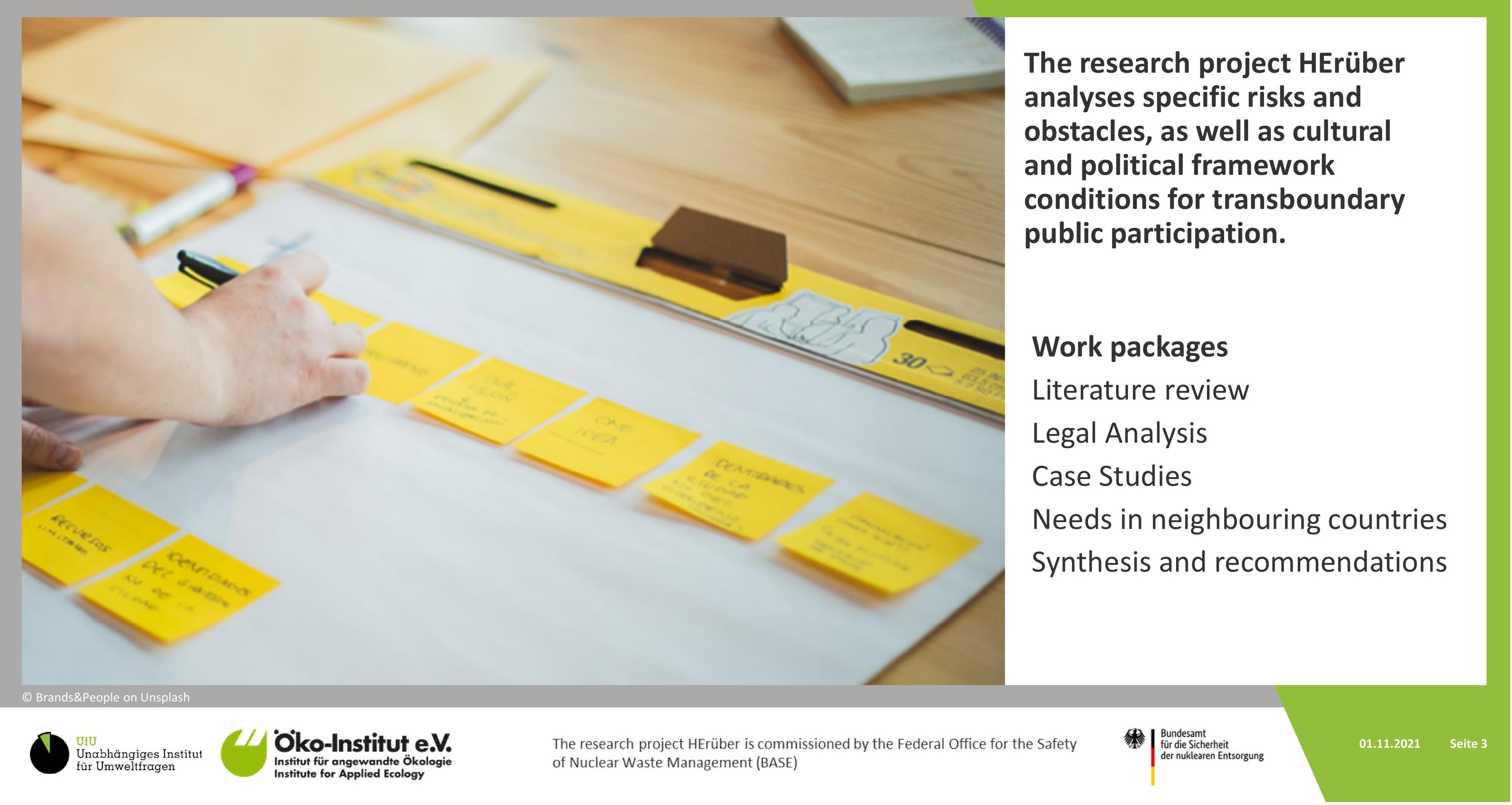




\section{Challenges for cross-border public participation procedures}

Risks and obstacles of public

participation in the search for a

nuclear repository

- Acceptance of the search procedure is a prerequisite for the acceptability of the repository site

- Complexity

- Constellations of actors

- Lack of knowledge and knowledge asymmetries

- Politicisation / Conflict
Risks and obstacles of

transboundary public

\section{participation}

- Unequal opportunities for foreign and domestic stakeholders

- Procedural uncertainties

- High time and resource requirements

- Language barriers
- Different competences in transboundary public participation and interdependencies with regional competences and networks

- Low interest of the foreign public 


\section{Success factors for...}

... public participation in the search for a nuclear repository

- Ensuring fairness of the procedure

- Countering complexity

- Deal with changing actors constellations
... transboundary public

participation

- Clarity of the process / Good explanation of the process, roles, tasks and responsibilities

- Early participation for all

- Planning appropriate resources and flexibility

- Quality translation and interpretation
- Use of clear language, detailed graphics and maps

- Willingness to communicate and cooperate

- Sensitive handling of intercultural differences

- Measures to activate the foreign public 


\section{Specific cultural and political framework conditions in neighbouring countries}

- Political framework = democratic structure, legal prerequisites and institutional setting

- Cultural framework = norms, traditions, meanings and attitudes of different social groups in a specific region or national context

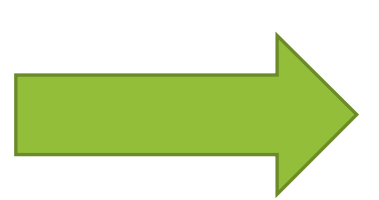

Neighbouring countries differ as well regarding collaborative decision making processes $\rightarrow$ long tradition vs. no or little experience

National contexts differ internal, e.g. with regard to participation and attitudes towards nuclear facilities 


\section{Influence of political culture on participation}

\section{Five dimensions of culture}

(Source: https://geerthofstede.com/; Enserink et al. 2007)

Relevance for participation:

$\rightarrow$ high power distance $=$ rather top-down decision making

$\rightarrow$ collective orientation $=$ more participatory processes

$\rightarrow$ traditional role models $=$ less participation

$\rightarrow$ strong catholic influence $=$ less participation

Power

distance 


\section{Neighbouring countries with high requirements for participation and rather critical attitude towards nuclear facilites}

\begin{tabular}{|c|c|c|c|c|}
\hline & Denmark & Netherlands & Austria & Switzerland \\
\hline $\begin{array}{l}\text { participation } \\
\text { experience }\end{array}$ & $\begin{array}{l}\text { long tradition in } \\
\text { participatory processes } \\
\text { (e.g. consensus } \\
\text { conferences); role models } \\
\text { not so important }\end{array}$ & $\begin{array}{l}\text { long tradition in early } \\
\text { stakeholder-participation } \\
\text { with consensus orientation; } \\
\text { role models not so } \\
\text { important; power distance } \\
\text { little }\end{array}$ & $\begin{array}{l}\text { lots of guidelines for } \\
\text { participatory } \\
\text { processes, some direct } \\
\text { democratic elements } \\
\text { institutionalised }\end{array}$ & $\begin{array}{l}\text { half-direct democracy } \\
\text { model with plebiscites } \\
\text { on all political levels; } \\
\text { early information of } \\
\text { public and referenda } \\
\text { common }\end{array}$ \\
\hline $\begin{array}{l}\text { attitude } \\
\text { towards } \\
\text { nuclear } \\
\text { facilities }\end{array}$ & $\begin{array}{l}\text { early societal resistance } \\
\text { against nuclear energy, } \\
\text { three decommissioned } \\
\text { research reactors, no } \\
\text { commercial reactor }\end{array}$ & $\begin{array}{l}\text { early and strong societal } \\
\text { resistance and rather } \\
\text { negative attitude towards } \\
\text { nuclear facilities; small- } \\
\text { scale nuclear programme }\end{array}$ & $\begin{array}{l}\text { high risk perception of } \\
\text { and strong opposition } \\
\text { towards nuclear } \\
\text { facilities; no } \\
\text { commercial and only }\end{array}$ & $\begin{array}{l}\text { ambivalent attitude } \\
\text { towards nuclear } \\
\text { facilities; five } \\
\text { commercial reactors, } \\
\text { but decision to opt out }\end{array}$ \\
\hline
\end{tabular}




\section{Neighbouring countries with diverse participation experiences and rather positive attitude} towards nuclear facilites

\begin{tabular}{|c|c|c|c|c|}
\hline & France & Czech Republic & Poland & Belgium \\
\hline $\begin{array}{l}\text { participation } \\
\text { experience }\end{array}$ & $\begin{array}{l}\text { ambivalent: tradition of } \\
\text { social movements, at the } \\
\text { same time until 1980ies } \\
\text { centralized characteristic } \\
\text { and traditional top-down } \\
\text { decision-making (power } \\
\text { distance) }\end{array}$ & $\begin{array}{l}\text { still young } \\
\text { democracy (since } \\
\text { 1992) with little } \\
\text { importance of } \\
\text { participatory } \\
\text { elements (power } \\
\text { distance) }\end{array}$ & $\begin{array}{l}\text { strong role models and } \\
\text { high uncertainty } \\
\text { avoidance as well as } \\
\text { power distance; } \\
\text { participatory elements } \\
\text { less important }\end{array}$ & $\begin{array}{l}\text { high power distance and } \\
\text { uncertainty avoidance } \\
\text { and as well strong role } \\
\text { models; little } \\
\text { participation experience }\end{array}$ \\
\hline $\begin{array}{l}\text { attitude towards } \\
\text { nuclear facilities }\end{array}$ & $\begin{array}{l}\text { risk perception of nuclear } \\
\text { facilites less marked; } 58 \\
\text { commercial reactors }\end{array}$ & $\begin{array}{l}6 \text { commercial } \\
\text { reactors, rather } \\
\text { nuclear friendly } \\
\text { attitude, } \\
\text { oppositional groups } \\
\text { marginalized }\end{array}$ & $\begin{array}{l}\text { up to date no } \\
\text { commercial reactor; } \\
\text { regionally diverse } \\
\text { attitude (e.g. negative } \\
\text { in cross-border region } \\
\text { to Germany) }\end{array}$ & $\begin{array}{l}\text { ambivalent attitude and } \\
\text { ongoing controversy } \\
\text { regarding site selection; } \\
\text { risk perception less } \\
\text { marked; } 7 \text { commercial } \\
\text { reactors }\end{array}$ \\
\hline
\end{tabular}

\section{1.}




\section{Potential border regions}

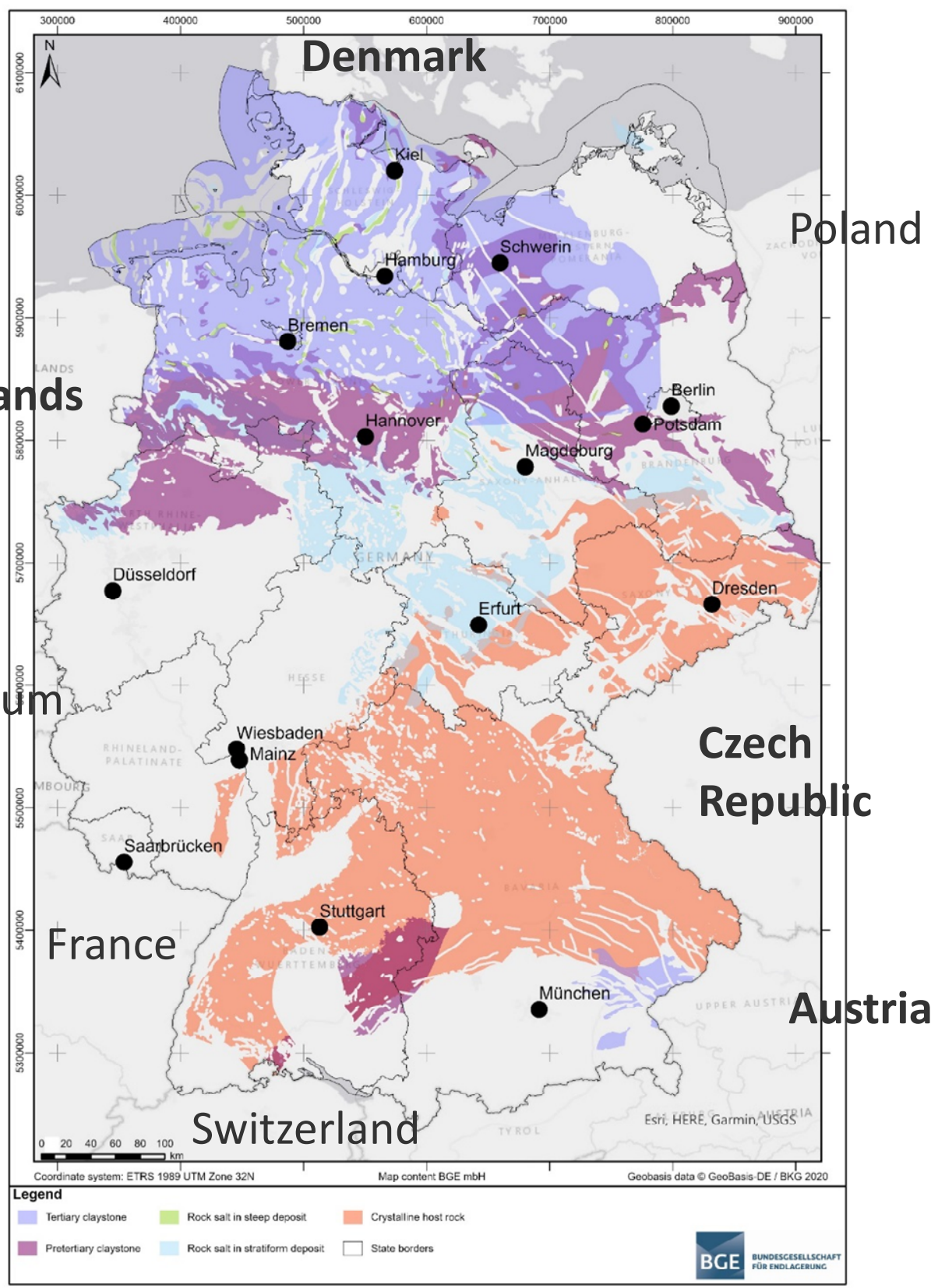




\section{Outlook}

Work package 3: exploration of challenges and success factors of crossborder participation towards three case studies

- Swiss planning procedure on deep geological disposal $(\mathrm{CH})$

- EIA for the extension of the nuclear power plants in Dukovany (CZ)

- SEA for the revision of Maritime spatial plans (DE)

Work package 5: requirements for cross-border participation in potential regions

- Five cross-border regions will be analysed regarding participation requirements 


\section{Thank you!}

\section{Contacts:}

Franziska Sperfeld

Dr. Melanie Mbah

franziska.sperfeld@ufu.de

m.mbah@oeko.de

All presented concepts, ideas and texts are intellectual property of UfU e.V. 